\title{
Conceptual Problems in Quantum Field Theory
}

\author{
Walter F. Wreszinski \\ Instituto de Física, Universidade de São Paulo, Caixa Postal 66318, 05315-970 São Paulo, Brazil
}

Received on 26 November, 2003

\begin{abstract}
We review some conceptual problems in quantum field theory, with an emphasis on exact results and open problems.
\end{abstract}

We review some conceptual problems in quantum field theory, with an emphasis on exact results and open problems. The review is organized as follows: 1 . The Stückelberg - Bogoliubov - Shirkov - Epstein - Glaser $S(g)$ operator. A brief review of perturbative results, including the adiabatic limit. Open problems: the infrared problem in QCD and its relation to gauge invariance. The problem of convergence and asymptotic character of the $S$-matrix series: the problem of large coupling. Problems with the series for (large and) small coupling: definition and triviality.
2. Nonperturbative results and problems: Triviality of the $S$ matrix and nonperturbative construction of $S(g)$. Open problems.

\section{Stückelberg-Bogoliubov-Shirkov- Epstein-Glaser $S(g)$ operator.}

In perturbation theory $S(g)$ is the generating functional for the time-ordered products of Wick polynomials,

$$
S(g)=1+\sum_{n=1}^{\infty} \frac{1}{n !} \int d^{4} x_{1} \ldots d^{4} x_{n} T_{n}\left(x_{1}, \ldots, x_{n}\right) \cdot g\left(x_{1}\right) \cdot\left(g_{2}\right) \ldots g\left(x_{n}\right),
$$

where $g \in \mathcal{D}\left(\mathbb{R}^{4}\right)$ is the "adiabatic switching".

The $n$-point operator-valued distributions $T_{n}$ are the basic objects of the theory. They are constructed inductively from $T_{1}$, through causality, unitarity and Lorentz-covariance:

a) $S(g)^{-1}=S(g)^{*} \quad$ (unitary)

b) $S\left(g_{1}+g_{2}\right)=S\left(g_{1}\right) S\left(g_{2}\right)$ if

a. $\operatorname{supp}_{1}>\operatorname{supp} g_{2} \quad$ (this ordering refers to the timecoordinate)

b. $\operatorname{supp}_{1} \sim \operatorname{supp} g_{2} \quad$ (causality)

Where " $\sim$ " means spaceline;

c. There exists a unitary representation $U(a, \wedge)$ of the Poincaré group $P_{+}^{\uparrow}$ on Fock space $\mathcal{F}$ (the free field representation) such that (Poincaré covariance)

$$
U(a, \wedge) S(g) U(a, \wedge)^{-1}=S(\{a, \wedge\} g)
$$

Above, supp $g$ means the (space-time) support of $g$, assumed to be an infinitely differentiable function of compact support, a class denoted by $\mathcal{D}\left(\mathbb{R}^{4}\right)$ - and

$$
(\{a, \wedge\} g)(x) \equiv g\left(\wedge^{-1}(x-a)\right) .
$$

What are the advantages of working with $S(g)$ ?

Less is required than the Wightman axioms; for $g \in \mathcal{D}$, we may work in Fock space, the infrared problem is decoupled and arises only in the adiabatic limit $g \rightarrow 1$. However: the main properties of general quantum field theory are preserved, in contrast to "effective" field theories.

Let now $T_{1}(x)$ be the interaction Lagrangian in first order (e.g. $T_{1}(x)=$ ie $: \bar{\psi}(x) \gamma^{u} \psi(x): A_{\mu}(x)$ for QED: all fields free fields on $\mathcal{F}$ ). It turns out that by causality

$$
T_{n}\left(x_{1}, \ldots, x_{n}\right)=T\left(T_{1}\left(x_{1}\right) \ldots T_{i}\left(x_{n}\right)\right)
$$

away from the total diagonal

$\Delta_{n}\left(x_{1}, \ldots, x_{n}\right)=\left\{\left(x_{1}, \ldots, x_{n}\right)\left(x_{1}=x_{2}=\ldots=x_{n}\right)\right\}$.

The extension of the $T\left(x_{1}, \ldots, x_{n}\right)$ to the total diagonal is not unique and is fixed by Poincaré covariance, unitarity and (re) normalizability: the scaling degree of each local term, obtained by normalizing $T_{n}$, is $\leq 4$.

This scaling degree - also called Steinmann degree is defined in the process of distribution splitting, a basic 
method used in causal perturbation theory ([1-3]), which is auxiliary in the determination of $T_{n}$. A convenient summary may be found in [4]. This degree is related to Weinberg's power counting [11]. Together with an additional requirement - perturbative quantum gauge invariance - the $T_{n}$ are determined also in nonabelian gauge theories, but the "gauge group" - with antissymetric structure constants - is derived from the above axioms [3]. It is to be emphasized that the requirement of perturbative quantum gauge invariance is purely quantum, without any reference to classical physics. It involves a gauge charge $Q$ :

$$
A^{\prime} \mu(x)=e^{-i \lambda Q} A^{\mu}(x) e^{i \lambda Q}
$$

wich may be expanded by means of the Lie series $A^{\mu}(x)-$ $i^{\lambda}\left[Q, A^{\mu}(x)\right]+0\left(\lambda^{2}\right)$, where $A^{\mu}(x)$ is a free (massive or massless) vector field in the theory. Comparing with the desired expansion $A^{\prime \mu}(x)=A^{\mu}(x)+\lambda \partial^{\mu} u(x)+0\left(\lambda^{2}\right)$, which is suggested by classical field theory, except that now $u(x)$ is, naturally, assumed to be a free quantum field with the same mass as $A^{\mu}$, we conclude that

$$
\left[Q, A^{\mu}(x)\right]=i \partial^{\mu} u(x)
$$

which determines $Q$ up to a $c$-number, due to the irreducibility of the Fock representation. It turns out [3] that $u(x)$ is a Fermionic ghost field, in order that $Q$ have the important property of being nilpotent:

$$
Q^{2}=0
$$

As remarked, the method also works (with simplifications) for massive gauge theories [6]. Quantize the free vector fields $\left(A_{a}^{\mu}\right)_{a=1, \ldots, M}$ by

$$
\left(\square+m_{a}^{2}\right) A_{\mu}^{a}=0 ;\left[A_{a}^{\mu}(x), A_{b}^{\nu}(y)\right]=i g^{\mu \nu} \delta_{a, b} \Delta_{m}(x-y), \quad A_{a}^{\mu *}=A_{a}^{\mu},
$$

where $\Delta_{m}$ is the Pauli-Jordan distribution of mass $m$, which corresponds to the Feynman gauge. The representation of this *-algebra requires an indefinite inner product space. It is thus natural to work in a Krein Fock space $\mathcal{F}$, denoting the usual scalar product in $\mathcal{F}$ by $(\cdot, \cdot)$ and $A^{+}$the adjoint of $A$ with respect to $(\cdot, \cdot)$. Let $J$ be the Krein operator: $J^{2}=1 ; J^{+}=J$. Then the indefinite inner product $\langle\cdot, \cdot\rangle$ is defined by

$$
\langle\psi, \phi\rangle \equiv(\psi, J \phi) \quad \psi, \phi \in \mathcal{F}
$$

and $*$ denotes the adjoint with respect to $\langle\cdot, \cdot\rangle$ :

$$
0^{*}=J 0^{+} J ;\langle 0 \psi, \phi\rangle=\left\langle\psi, 0^{*} \Phi\right\rangle \text {. }
$$

For the ghost fields, $u_{a}(x)^{*}=u_{a}(x)$ and for the antighost fields $\widetilde{u}_{a}(x)\left(\neq u_{a}^{+}(x) !\right), \widetilde{u}_{a}^{*}(x)=-\widetilde{u}_{a}(x)$ ([3], pg. 126). Convergence of the integral for $Q$ (which results from (4)),

$$
Q=\int d^{3} x \sum_{a} \partial_{\nu} A_{a}^{\nu}(x) \vec{\partial}_{0} u_{a}(x)
$$

(massless case) follows by a method due to Requardt [19]. Notice that by $A_{a}^{\nu *}=A_{a}^{\nu}$ and $u_{a}(x)^{*}=u_{a}(x), Q$ above is an unbounded $*$-symmetrical nilpotent operator on $\mathcal{F}$ :

$$
Q=Q^{*} \quad(\text { on a dense invariant domain } \mathcal{D})
$$

This structure implies perturbative pseudo unitarity in the Krein-Fock Hilbert space, defined as being the following property

$$
T_{n}\left(x_{1}, \ldots, x_{n}\right)^{*}=\widetilde{T}_{n}\left(x_{1}, \ldots, x_{n}\right)
$$

where the $\widetilde{T}_{n}\left(x_{1}, \ldots, x_{n}\right)$ are the $n$-point distributions corresponding, however, to $S(g)^{-1}$ instead of $S(g)$ ([3], pg. 127). By means of (5) one easily finds [3] that

$$
\mathcal{D}=\operatorname{ran} Q \oplus\left(\operatorname{ker} Q \bigcap \operatorname{ker} Q^{+}\right) \oplus \operatorname{ran} Q^{+} .
$$

In addition it is assumed that

$$
J_{\text {ker } Q^{+}} \cap \operatorname{ker} Q=\mathbf{1} \quad \text { (positivity assumption). }
$$

Then the $\langle\cdot, \cdot\rangle-$ product is positive definite on

$$
\mathcal{H}_{\text {phys }}=\operatorname{ker} Q \bigcap \operatorname{ker} Q^{+}=\operatorname{ker} Q / \overline{\operatorname{Ran} Q},
$$

which is interpreted as the physical subspace of $\mathcal{F}$ : it does not contain the unphysical degrees of freedom [3]. From this and (10) the usual unitarity a) on Hphys follows ([3], pg. 128). This structure, with the Hermiticity condition (9) is most clearly expounded in [6], (which we followed), in which it is also shown that for massive theories the condition of perturbative quantum gauge invariance may be replaced by the physical consistency of the $S$ matrix

$$
[Q, S] / \operatorname{ker} Q=0,
$$

where $S$ - the true $S$ matrix - is the adiabatic limit of $S(g)$

$$
S \psi=\lim _{\in \rightarrow 0} S\left(g_{\in}\right) \psi
$$

for all $\psi \in \mathcal{D}$, where $g_{0} \in \mathcal{D}\left(\mathbb{R}^{4}\right)$, and

$$
g_{\in}(x) \equiv g_{0}(\in x) \text {, }
$$

where $g \equiv g_{0}(0)>0$ is the (physical) coupling constant. For massive theories the existence of the adiabatic limit was proved by Epstein and Glaser [7]. 
From theories with massless particles, a more elaborate condition is required ([3], pg. 99), which is equivalent to the Ward-Takahashi identity for QED ([3], pg. 100) and which, for massless $S U(N)$ - Yang-Mills theories, implies the Slavnov Taylor identities ([20], [16]).

The differences regarding the usual BRST charge are ([3], pg. 240): a) the BRST variation of the total Lagrangian is zero, but the gauge variation of the interaction $T_{1}$, only, is not zero but a divergence; b) the BRST transformation operates on the interacting fields whereas the gauge variation transforms free fields. See, however, recent more general formulations [5].

What are the advantages of causal perturbation theory? First and foremost is the treatment of gravity [3]: in the traditional approach, with ultraviolet regularization, it is unclear, due to non-renormalizability, how to obtain cutoffindependent finite results. In the causal theory one has only to fix finite (normalization) constants obtained in the process of distribution splitting. Of course, the number of finite constants grows with each order, and it is unclear how to fix them; but this (open) problem is of a less crucial nature. Secondly there is the conceptual clarity: for instance, the existence of a scalar field ("Higgs field") is also derived from the "axioms", without assuming spontaneous breakdown of symmetry. Since gauge symmetry is a local symmetry, it cannot be broken (Elitzur's theorem, see [8]), and it seems very artificial to introduce an extra global symmetry which is, then, broken. Finally, for some superrenormalizable theories, a mechanism of dynamical mass generation may take place, e.g., in $(\mathrm{QED})_{3}$. Because

$$
\pi_{\mu \nu}\left(k^{2}=0\right) \neq 0
$$

where $\pi_{\mu \nu}$ is the vacuum polarization tensor, the photon acquires a nonzero mass.

In the causal theory there is no regularization ambiguity for the photon mass [4], in contrast to the ambiguities found by Deser, Jackiw and Templeton in [9].

As remarked the true scattering matrix is recovered only in the adiabatic limit $g \rightarrow 1$ which was shown in [7] for massive theories, and in [10] for QED (see also [11] for an approach in terms of the algebra of observables). In QED there exists the Bloch-Nordsieck cancellation, but the latter is not effective in non abelian gauge theories. For theories such as QED, if we call $F_{n}$ the amplitude for a given process, with the additional production of $n$ ("soft") photons ("Brehmsstrahlung"), the overall (inclusive) physical probability

$$
P=\sum_{n}\left|F_{n}\right|^{2}
$$

is infrared finite. In QED we also have exponentiation of infrared divergences: in any $S$-matrix amplitude $F_{0}$, the infrared divergences may be factored out,

$$
F_{0}=e^{D} G_{0},
$$

where $D$ is a simple explicit divergent quantity, and $G_{0}$ a new pertubative series, which is free of infrared divergences.
It seems that in QCD there is no cancellation, but exponentiation holds. Thus $D=-\infty, \quad F_{0}$ vanishes. Since there is no cancellation, physical probabilities vanish as well - as far as perturbation theory is, at least, asymptotic - and one speaks of perturbative infrared slavery. This is an unproved conjecture for $\mathrm{qcd}$, at least for initial states which are not colour singlets; see [12]. In refs. [13] and [14] the authors start with the remark that individual Feynman graphs, are not gauge invariant, but that appropriate sums ("invariance classes"), are: for instance, for second-order Compton scattering, the sum of the two diagrams where the external photon lines are interchanged.

They generate these invariance classes in QED by looking at Green's functions of a "dressed" electron field $\chi$,

$$
\chi=e^{\int f^{\mu} A_{\mu} \tau} \psi,
$$

where $\tau$ is a translation operator $\tau_{p^{\prime}} \rightarrow p^{\prime}-k^{\prime}$ acting on momentum space, $f^{\mu}$ is a chosen function and $\psi$ is the usual electron field, thus given by the nonlocal transformation (the inverse of (19)):

$$
\psi\left(p^{\prime}\right)=\int d^{4} q^{\prime} S\left(p^{\prime}, q^{\prime}, A_{\mu}\right) \chi\left(q^{\prime}\right)
$$

with

$$
\begin{aligned}
S\left(p^{\prime}, q^{\prime}, A_{\mu}\right)= & \exp \left[-e \int d^{4} k f_{\mu}\left(q^{\prime}, k\right) A^{\mu}(k) \cdot \tau\right] \\
& \times \delta\left(p^{\prime}-q^{\prime}\right) .
\end{aligned}
$$

With this manifestly gauge-invariant formulation of QED there follows simple proof of infrared exponentiation.

In qcd, due to the self-coupling of the gluons, the gluon field must be dressed too. This is an open problem which affects the whole structure of qcd. Indeed, the "ellipsoidal bound" on the Gribov horizon contradicts the asymptotic behaviour at large momentum predicted by the perturbative renormalization group [21]. The relation between the infrared problem and gauge invariance remains thus obscure in these theories, and the limit $g \rightarrow 1$ remains open.

In addition to ultraviolet and infrared divergences, there arises an independent divergence in quantum field theory, that of the (already renormalized) $S$-matrix series

$$
S(g=\alpha)=\sum_{n=0}^{\infty} S_{n} \alpha^{n}
$$

We review this aspect briefly following [15]. Typically, $S_{n} \sim(n !)^{L}$ for some $L$ (e.g. $L=1,2, \ldots$ ) due to the growth of the number of graphs at each order $n$. Thus, $S(\alpha)$ is a divergent series, but which is believed to be asymptotic, e.g., for the gyromagnetic ratio (replacing $S(\alpha))$ :

$$
\left(\frac{g-2}{2}\right)(\alpha)=\frac{1}{2} \frac{\alpha}{\pi}-0.328\left(\frac{\alpha}{\pi}\right)^{2}+0\left(\alpha^{6}\right)
$$


where

$$
\alpha=\text { fine structure constant }=\frac{1}{137.0 \ldots} .
$$

Given a function $f$ defined on an open interval $a<x<b$, one says that the function $f_{N}$ defined on the interval, is asymptotic to $f$ of order $N$ at $a$ if $\left(x \rightarrow a_{+}\right.$means the limit from the right):

$$
\lim _{x \rightarrow a_{+}} \frac{f(x)-f_{N}(x)}{(x-a)^{N}}=0 .
$$

This general definition applies in particular to the expression

$$
f_{N}(x)=\sum_{n=0}^{N} a_{n}(x-a)^{n}
$$

If one is given a sequence $\left\{a_{0}, a_{1}, a_{2}, \ldots\right\}$ and
$\sum_{n=0}^{N} a_{n}(x-a)^{n}$ is asymptotic to $f$ of order $N$ at
$a$ for all $N=0,1,2, \ldots$ then one says that the series $\sum_{n=0}^{\infty} a_{n}(x-a)^{n}$ is asymptotic to $f$ at a and writes

$$
f(x) \sim \sum_{x=0}^{\infty} a_{n}(x-a)^{n}
$$

Now, if $\lim _{x \rightarrow a}\left[f(x)-f_{N}(x)\right][x-a]^{-N}=0$, it evidently follows that $\lim _{x-a}\left[f(x)-f_{N}(x)\right][x-a]^{-k}=0$ for $k=0,1, \ldots, N-1$, so when $\sum_{n=0}^{N} a_{n}(x-a)^{n}$ is asymptotic to $f$ of order $N$ at $a$, then:

$$
\begin{aligned}
& \lim _{x \rightarrow a_{+}} f(x)=a_{0}-f(a+), \\
& \lim _{x \rightarrow a_{+}}[f(x)-f(a+)][x-a]^{-1}=a_{1}=f^{\prime}(a+), \\
& \quad \vdots \\
& \lim _{x \rightarrow a_{+}}\left[f(x)-\sum_{n=0}^{N-1} \frac{f^{(n)}(a+)}{n !}(x-a)^{n}\right][x-a]^{-N}=a_{N}=\frac{f^{(N)}(a+)}{N !} .
\end{aligned}
$$

Thus, when a function $f$ is asymptotic of order $N$ at $a$ to an expression $\sum_{n=0}^{N} a_{n}(x-a)^{n}$, the function $f$ necessarily has $N$ derivatives from the right at $a$ and the coefficients $a_{n}$ are uniquely determined as Taylor coefficients

$$
a_{n}=\frac{f^{n}(a+)}{n !}, \quad n=0,1, \ldots, N
$$

Thus, the assertion that the gyromagnetic anomaly $\left(\frac{g-2}{2}\right)(\alpha)$ has the asymptotic series $\sum_{n=1}^{\infty}$ an $(\alpha / \pi)^{n}$ at zero means no more and no less than that $\left(\frac{g-2}{2}\right)(\alpha)$ is defined for $\alpha$ in some interval $a<\alpha<\alpha_{0}$ and has derivatives of all orders from the right at 0 .

Now, for QED, $\alpha \approx 10^{-2}$. Suppose now that $S_{n} \simeq$ $c n ! \quad c>0$. Consider $S_{N-1}(\alpha)=\sum_{n=0}^{N-1} S_{n} \alpha^{n}$ asymptotic to $S(\alpha)$ at $\alpha=0$. Thus, by Taylor's formula with remainder

$$
\begin{aligned}
& S(\alpha)-S_{N-1}(\alpha)=S(\alpha)-\sum_{n=0}^{N-1} \frac{S^{(n)}(0+)}{n !} \alpha^{n}= \\
& =R_{N}(\alpha) ; R_{N}(\alpha)=\int_{0}^{\alpha} \frac{S^{(N)}(t)}{(N-1) !}(\alpha-t)^{N-1} d t .
\end{aligned}
$$

Now $R_{N}(\alpha) \underset{N \rightarrow \infty}{\longrightarrow} \infty$ for any $\alpha \neq 0$ but $R_{N}(\alpha) \simeq$ $c N ! \alpha^{N}$. For $\alpha$ small enough we may consider (assuming $N$ large enough - Stirling's formula; treating $N$ as continuous parameter; justified a posteriori):

$$
\begin{aligned}
\ln R_{N}(\alpha) \sim & N \ln N+N \ln \alpha \\
\frac{d}{d N} \ln R_{N}(\alpha) & \cong 1+\ln N+\ln \alpha=0 \\
\Longrightarrow \ln N & =-1-\ln \alpha \Longrightarrow N=e^{-1} e^{-\ln \alpha} \\
& \simeq e^{3} \simeq 25 \text { for } \alpha \approx 10^{-2}, \\
\frac{d^{2}}{d N^{2}} \ln R_{N}(\alpha) & =\frac{1}{N}>0 \quad \text { (minimum!). }
\end{aligned}
$$


If, however, $\alpha \simeq 10$ (qcd) there is no minimum, so that, even if the series is asymptotic, there is no hope of approximating the true function by a finite sum of the perturbation series!

This is the problem of large coupling. It can only be attacked by a construction of the theory in an intrinsically nonperturbative way. A first step in this direction is reported in section 2.1 .

An alternative approach would be to to try to prove a property of the nonperturbative solution which guarantees that a special summability method (e.g. Borel's) applied to the perturbation series necessarily yields a unique answerthat is, the right one, see [15] for a nice review.

A second nonperturbative issue is the triviality of the $S$ matrix. It is conjectured [16] that the $\left(: \phi^{4}:\right)_{4}$ or (QED) theories, being not asymptotically free [16], lead to a trivial $S$ matrix, i.e., $S=\mathbf{1}$. This is sometimes taken as an argument in favor of "effective theories": since the photon has a hadronic component with a probability $\alpha \sim \frac{1}{137} \sim 1 \%$, QED is not an isolated field of physics but is intimately linked to strong interactions. This is physically clear, but, simply theoretically, what is then the renormalized (Dyson) $S$-matrix of $(\mathrm{QED})_{4}$ ?

It cannot be asymptotic if it is trivial, and yet it yields the best and most precise results of science! Weinberg [16] argues that the rigorous lattice-field theory results of Aizenman-Fröhlich are not conclusive to show triviality of $\left(: \phi^{4}:\right)_{4}$, as well as that the renormalization-group arguments are inconclusive to show triviality of $\left(: \phi^{4}:\right)_{4}$ and $(\mathrm{QED})_{4}$. Nevertheless triviality might be true, at least for $\left(: \phi^{4}:\right)_{4}$ ! A partial result in this direction is in section 2.2.

\section{Nonperturbative Results and Pro- blems}

\subsection{Nonperturbative construction of $S(g)$ (with L.A. Manzoni and O. Bolina)}

Let $H_{0}=\int \omega(k) a^{*}(k) a(k) d k$, with $\omega(k)=$ $\left(k^{2}+m^{2}\right)^{1 / 2}$, be the free field Hamiltonian corresponding to a zero-time scalar field $\phi(x, 0)$ of mass $m>0$, and, formally for $g \in \mathcal{D}\left(\mathbb{R}^{2}\right)$,

$$
\begin{aligned}
& V_{g}(t) \equiv \int d x g(x, t): \phi^{4}(x, 0):, \\
& H_{g}(t) \equiv H_{0}+V_{g}(t) \\
& \widetilde{H}(t) \equiv H_{g}(t)+M 1 .
\end{aligned}
$$

( $M$ a constant introduced to make $\widetilde{H}(t)$ a positive operator). Then (W.W., L.A. Manzoni, O. Bolina, preprint, Univ. São Paulo, to appear in J. Math. Phys.):

THEOREM There exists a unique solution of the evolution equation

$$
i \frac{\partial u(t, s)}{\partial t} \psi=\widetilde{H}(t) U(t, s) \psi
$$

where $\psi \in D(\widetilde{H}(s))$ is dense in $\mathcal{F}$. Further, defining the "Dirac picture propagator" by

$$
U^{D}(t, s) \equiv e^{i\left(H_{0}+M\right) t} U(t, s) e^{-i\left(H_{0}+M\right)_{s}},
$$

then $(s-\lim$ means the strong limit, i.e., on every vector $\phi$ of $\mathcal{F}$ ),

$$
S(g) \equiv \underset{\substack{t \rightarrow+\infty \\ s \rightarrow-\infty}}{s-\lim ^{D} U^{D}(t, s)}
$$

exists and satisfies the unitary a) and first causality condition b) (for supp $g_{1}>\operatorname{supp}_{2}$ ).

The second causality condition and Lorentz covariance are still open, as well as the adiabatic limit along the lines of [7] or [11].

\subsection{Triviality of the $S$ Matrix}

There are virtually no results on the issue of triviality of the $S$ matrix. $K$ Hepp [17] studied some models which have no vacuum polarization (a persistent vacuum), but nontrivial renormalization of the mass and the coupling constant. He called these Lee models, and this class of (partially soluble) models the "Royaume Intermédiaire".

Let $H_{0}$ denote the free Hamiltonian of scalar Bosons of mass $m>0$,

$$
B_{\epsilon}=[-\pi / \epsilon, \pi / \epsilon]^{s}, \quad \text { with } \quad \epsilon>0 \quad ;
$$

$\epsilon^{-1}$ plays the role of a (momentum) cutoff, which later tends to infinity in an appropriate manner ([17], [18]). The Hamiltonian is:

$$
H_{\epsilon}=H_{0}+V
$$

where

$$
V_{\epsilon}=\lambda \int_{B_{\epsilon}} \prod_{i=1}^{4} \int \frac{d \vec{p}_{i}}{\sqrt{\mu_{i}}} \delta\left(\vec{p}_{1}+\vec{p}_{2}-\vec{p}_{3}-\vec{p}_{4}\right) a^{*}\left(\vec{p}_{1}\right) a^{*}\left(\vec{p}_{2}\right) a\left(\vec{p}_{3}\right) a\left(\vec{p}_{4}\right),
$$


and

$$
\mu_{i}=\mu\left(\vec{p}_{i}\right)=\sqrt{\vec{p}_{i}^{2}+m^{2}}, \quad m>0 .
$$

The number operator commutes with $H$ and Fock space decomposes into (labelled by $N$ ) dynamically independent sectors, with no vacuum polarization (but: renormalization of mass and coupling constant are necessary!)

There are the following results [18]; $s$ is given by (36)!

THEOREM [18]

a) If $s=3$, either the theory is trivial or $E_{0}(N) \leq$ $-B N^{2}$, with $B>0$ independent of $N$, and $E_{0}(N)$ the ground state energy in the sector of $N$ particles (thus, in this case the theory is physically inacceptable, because it leads to infinite energy density);

b) If $s=2$, the theory is nontrivial.

To the extent that (37), (38) is an approximation of $\left(: \phi^{4}:\right)_{s+1}$ (discussed in [18]), the previous theorem shows the marked difference between $s=2(s+1=3)$ and $s=3(s+1=4)$, which seems to support the triviality conjecture. Much remains to be done concerning this important issue!

\section{Acknowledgement}

The author is grateful to Prof. P. van Nieuwenhuizen for raising several questions on the causal theory, as well as instructive comments.

\section{REFERENCES}

[1] H. Epstein and V. Glaser, Ann. Inst. Henri Poincaré A19, 211 (1973).

[2] G. Scharf, Finite Quantum Electrodynamics: the Causal Approach Second edition - Springer, 1995.

[3] G. Scharf, Quantum Gauge Theories, A True Ghost Story - Wiley 2001.
[4] G. Scharf, W.F. Wreszinski, B.M. Pimentel, and J.L. Tomazelli, Ann. Phys. 231, 185 (1994).

[5] N. Nakanishi and I. Ojima, Covariant Operator Formalism of Gauge Theories and Quantum Gravity, World Scientific Lect. Notes in Phys. vol. 27.

[6] M. Dütsch and B. Schroer, J. Phys, A33, 4317 (2000).

[7] H. Epstein and V. Glaser in Renormalization Theory, ed. by G. Velo and A.S. Wightman, pp. 193-254 (1976).

[8] S. Elitzur, Phys. Rev. D12, 3978 (1975).

[9] S. Deser, R. Jackiw, and S. Templeton, Ann. Phys. 140, 372 (1982).

[10] Ph. Blanchard and R. Séneor, Ann. Inst. Henri Poincaré A23, 147 (1975).

[11] M. Dütsch and K. Fredenhagen, Comm. Math. Phys. 203, 71 (1999).

[12] R. Doria, J. Frenkel, and J.C. Taylor, Nucl. Phys. B168, 73 (1980).

[13] M. Bergére and L. Szymanovski, Phys. Rev. D26, 3350 (1982).

[14] D. Rello, Phys. Rev. D29, 2282 (1984).

[15] A.S. Wightman, Should We Believe in Quantum Field Theory? Cargése Lectures, 1984.

[16] S. Weinberg, The Quantum Theory of Fields, vol II Cambridge University Press 1996.

[17] K. Hepp, Théorie de la Rénormalisation Springer 1969.

[18] F.A.B. Coutinho, J.F. Perez, and W.F. Wreszinski, Ann. Phys. 277, 94 (1999).

[19] M. Requardt, Comm. Math. Phys. 50, 259 (1976).

[20] M. Dütsch, Inst. J. Mod. Phys. A12, 3205 (1997).

[21] G. F. Dell'Antonio and D. Zwanziger, Nucl. Phys. B 326, 333 (1989). 